\title{
Uma Análise Competitiva entre as Tecnologias Blockchain e Tangle para o Projeto de Aplicações IoT
}

\author{
Rafael Zerbini Alves da Mata ${ }^{1}$, Carlo Kleber da Silva Rodrigues ${ }^{2}$
}

${ }^{1}$ Faculdade de Tecnologia - Departamento de Engenharia Elétrica - Universidade de Brasília (UnB) - Brasília - DF - Brasil.

${ }^{2}$ Centro de Matemática, Computação e Cognição - Universidade Federal do ABC (UFABC) - Santo André - SP - Brasil.

\{rafael.zerbinia, carlokleber\}@gmail.com

\begin{abstract}
This paper aims at performing a competitive analysis between the Blockchain and Tangle technologies, mainly focusing on the design of IoT applications. To this end, we firstly carry out a theoretical comparative study of the data structures and algorithms used to manipulate information under each technology. Then, through simulations, we analyze different scenarios to assess the system security level in terms of data inviolability. Final results allow us to conjecture that Tangle is a more promising choice, besides constituting worthy insights for real designs of IoT applications. General conclusions and future work conclude this article.
\end{abstract}

Resumo. Este artigo tem por objetivo realizar uma análise competitiva entre as tecnologias Blockchain e Tangle, visando principalmente ao projeto de aplicações IoT. Para tanto, inicialmente é feito um estudo teórico comparativo das estruturas de dados e algoritmos usados para manipular informações sob cada tecnologia. Em seguida, por meio de simulação, diferentes cenários são analisados para aferir o nível de segurança do sistema em termos da inviolabilidade das informações, considerando o emprego de cada tecnologia. Os resultados finais permitem conjecturar que Tangle é uma alternativa mais promissora, além de se constituírem em subsídios para possíveis projetos de aplicações IoT. Conclusões gerais e trabalhos futuros encerram este artigo.

\section{Introdução}

A Internet das Coisas (do inglês, Internet of Things - IoT) possui diversas aplicações, incluindo redes inteligentes, cidades inteligentes [Gaur et al. 2015], contratos inteligentes e gestão de saúde [Hassanalieragh et al. 2015]. Neste cenário, a rede de comunicação subjacente deve oferecer processamento e disseminação de dados de maneira transparente, intensa e pervasiva, o que traz preocupações relacionadas a aspectos de segurança, privacidade e escalabilidade [Dorri; Kanhere; e Jurdak 2017].

Considere, por exemplo, uma operação de crédito entre dispositivos IoT regulada por um contrato inteligente em uma rede de comunicação peer-to-peer (P2P). Os nós dos dispositivos IoT armazenam cópias consistentes do contrato inteligente. Um dos dispositivos pode solicitar a atualização do estado do contrato para atribuir crédito a um outro dispositivo para prestação de um serviço. Os nós constituintes da rede são 
informados dessa atualização quando recebem individualmente o novo estado do contrato. Eventualmente, o dispositivo IoT destinatário do crédito verifica esse estado em seu nó e, em seguida, libera o serviço para o dispositivo solicitante.

Esse processo permite a troca de crédito na ausência de confiança mútua, uma vez que a transação é certificada por um terceiro, ou seja, a rede de nós, e é dificilmente reversível. O objetivo desse processo é, sobretudo, garantir que cada dispositivo observe o mesmo estado do contrato inteligente. A questão crucial de projeto é então fazer com que cada dispositivo permaneça sincronizado com a versão mais recente do contrato, além de garantir segurança, privacidade e escalabilidade.

Independentemente da natureza da aplicação IoT, a solução da questão ilustrada acima reside na escolha da tecnologia para manipular as informações das transações entre os dispositivos comunicantes. Nesse sentido, Blockchain [Nakamoto 2008] e Tangle [Popov 2017] são duas importantes tecnologias merecedoras de atenção na literatura [Dorri; Kanhere; e Jurdak 2017; Danzi et. al. 2017].

Blockchain se baseia na implementação de uma lista encadeada de blocos de transações (do inglês, blockchain). Esses blocos são adicionados à lista conforme são validados por meio de um processo matemático. Por sua vez, Tangle se baseia na implementação de um Grafo Acíclico Direcionado (do inglês, Directed Acyclic Graph DAG), onde cada nó é uma transação. Para que a transação seja adicionada ao DAG, há um processo matemático, como na Blockchain, e ainda ocorre a validação de duas outras transações pré-existentes no DAG.

Este contexto é a motivação para este artigo, cujo objetivo é realizar uma análise competitiva entre as tecnologias Blockchain e Tangle, visando ao desenvolvimento de possíveis projetos de aplicações IoT. Para este fim, é feito inicialmente um estudo teórico das estruturas de dados e algoritmos usados para manipular informações sob cada tecnologia, buscando avaliar as complexidades de tempo com respeito a operações de consulta e adição de transações. Em seguida, por meio de simulação, diferentes cenários de operação são analisados para mensurar o nível de segurança sistêmica, em termos da inviolabilidade das informações registradas.

Ante o objetivo anunciado, este artigo tem, como principal contribuição, a disponibilização de um único texto que provê um entendimento comparativo das tecnologias Blockchain e Tangle e, ainda, oferece uma análise competitiva entre as mesmas. Essa contribuição se constitui valiosa por prover subsídios relevantes para o desenvolvimento de possíveis projetos de aplicações IoT.

A organização do restante deste artigo é descrita a seguir. A Seção 2 apresenta as tecnologias Blockchain e Tangle. Os trabalhos relacionados são comentados na Seção 3. $\mathrm{Na}$ Seção 4, é realizado o estudo teórico das estruturas de dados e algoritmos utilizados nas tecnologias supracitadas. A Seção 5 analisa a segurança do sistema, com foco na garantia da inviolabilidade das informações. Por fim, conclusões gerais e trabalhos futuros aparecem na Seção 6. 


\section{Fundamentos}

\subsection{Tecnologia Blockchain}

Como mencionado, as transações dos clientes são agrupadas em blocos interligados formando uma lista encadeada, i.e., a blockchain. Cada bloco está ligado a apenas um bloco anterior a ele. Mineração é o processo matemático que precisa ser executado para que o bloco seja adicionado à blockchain. Esse processo é executado por um peer, denominado de minerador, ou por um grupo de peers, denominado de mining pool [Garay; Kiayias; e Leonardos 2015; Silva e Rodrigues 2016].

Os mineradores ou mining pools são também responsáveis por coletar as transações transmitidas em broadcast na rede e agrupá-las em blocos. Cada vez que um bloco é minerado, o correspondente minerador ou mining pool recebe uma recompensa, que é um incentivo financeiro para que a mineração seja continuamente realizada.

$\mathrm{Na}$ prática, a mineração consiste em tentativas sucessivas para determinar um valor de nonce que resolve o hash criptográfico do bloco de transações, atendendo a um certo critério. A dificuldade desse critério é ajustada com base na frequência com que os blocos são adicionados à blockchain. A determinação do nonce é a chamada prova de trabalho (do inglês, proof of work) de que o bloco foi verificado e pode ser adicionado. O algoritmo de hash criptográfico utilizado é o SHA256 [Gilbert e Handschuh 2004].

Além das transações, cada bloco possui um cabeçalho com metadados [Antonopoulos 2017]. A identificação de cada bloco é feita pelo hash criptográfico de seu cabeçalho. Como informado, cada bloco faz referência a apenas um bloco anterior, chamado de bloco pai. Essa referência é feita com o hash do cabeçalho do bloco pai. Ou seja, cada bloco contém o hash do cabeçalho de seu pai dentro de seu próprio cabeçalho.

A sequência de hashes que liga cada bloco ao seu pai cria uma cadeia de blocos que faz o caminho de volta até o primeiro bloco do sistema, denominado bloco gênese. A mudança da identidade de um bloco $B$ geraria um efeito cascata da mudança da identidade de todos os blocos subsequentes a ele. Esse efeito cascata constitui a base da segurança do sistema com relação à inviolabilidade das informações registradas, conforme explorado mais adiante na Seção 5.

A Blockchain teve sua origem em 2008, junto com a proposta da criptomoeda Bitcoin [Nakamoto 2008]. Apesar da concepção original para pagamentos eletrônicos, a Blockchain foi rapidamente identificada como de aplicabilidade mais abrangente [Dorri; Kanhere e Jurdak 2017; Huckle et al. 2016]. Por exemplo, na implementação de aplicações de controle de votos, de recursos em gestão orçamentária, de direitos de propriedades e, ainda, de comunicação em sistemas distribuídos, incluindo especialmente IoT [Dorri; Kanhere; e Jurdak 2017; Li e Liao 2017].

\subsection{Tecnologia Tangle}

A tecnologia Tangle surgiu para implementação da criptomoeda iota [IOTA 2017], então projetada em 2014 para a indústria de IoT. Sua aplicabilidade, no entanto, não se restringe a pagamentos eletrônicos, mas envolve um escopo bem mais abrangente, tal como ocorre com a tecnologia Blockchain. 
Como já mencionado, a tecnologia Tangle utiliza a estrutura DAG para armazenamento das transações. A função de hash criptográfico originalmente utilizada nessa tecnologia foi a Curl, tendo sido substituída mais tarde pela Keccak-384 [Sonstebo 2017], também conhecida como SHA-3 (Secure Hash Algorithm 3) [Chandrana e Manuelb 2016]. Os defensores dessa tecnologia professam que ela é a evolução da Blockchain, levando-se em conta os três aspectos comentados a seguir.

$\mathrm{O}$ primeiro aspecto diz respeito a não existência de recompensas na Tangle. $\mathrm{Na}$ Blockchain, a recompensa pode inviabilizar a execução de transações típicas de IoT. Isso porque essas transações tendem a ser de pequenos valores. Daí, a existência de uma recompensa de valor maior que o montante que está sendo transferido na transação não seria lógico. Simplesmente eliminar a recompensa na Blockchain tampouco seria aceitável, pois ela é o incentivo para a contínua mineração de blocos.

O segundo aspecto relaciona-se à homogeneidade funcional dos dispositivos IoT na tecnologia Tangle. Isto é, todos os participantes desempenham a mesma função. Isso não é observado na tecnologia Blockchain, pois há dois tipos distintos de participantes: aqueles que emitem transações e aqueles que aprovam transações.

Essa discriminação entre dispositivos pode levar a conflitos na mineração de blocos em virtude das recompensas previstas. Nesse caso, uma disputa entre mineradores ou mining pools pode transformar um paradigma de validação concebido originalmente descentralizado em um paradigma centralizado, constituído por mineradores ou mining pools de maior capacidade de processamento.

$\mathrm{O}$ terceiro aspecto refere-se à maior simplicidade da Tangle para adição de novas transações ao sistema. Essa adição envolve a resolução de uma prova de trabalho e a validação de duas transações pré-existentes. Ocorre que a prova de trabalho da Tangle, por considerar apenas uma transação, demanda um esforço computacional bem menor que àquele da mineração na Blockchain, que envolve múltiplas transações. Por sua vez, a validação de uma transação pré-existente é simples e pode ser realizada em tempo constante. A adição de transações é detalhadamente analisada na Subseção 4.2.

Para terminar esta subseção, cabe mencionar que as validações são representadas por arestas direcionadas no DAG, que partem da nova transação em direção às transações pré-existentes. Se não existe uma aresta direcionada entre uma transação $A$ e uma transação $B$, mas há um caminho direcionado entre elas, diz-se então que $A$ indiretamente aprova $B$. Quanto maior é o número de validações que uma transação possui, mais confiável é a validade dessa transação. Por definição, há ainda a transação denominada gênese, que representa a primeira transação do sistema e é validada direta ou indiretamente por todas as outras transações do DAG.

\section{Trabalhos Relacionados}

A tecnologia Blockchain possui um considerável número de trabalhos na literatura. $\mathrm{O}$ mesmo não pode ser afirmado para tecnologia Tangle, a qual é mais alvo de blogs e de outros veículos informais. Estudos comparativos entre essas duas tecnologias tampouco são fáceis de encontrar. Neste contexto, esta seção discorre sobre alguns dos mais recentes e relevantes trabalhos da literatura que contribuem para o objetivo deste artigo, buscando ofertar uma visão do estado da arte desta área de pesquisa. 
Opara e Soluade (2015) e Singh (2014) analisam ataques cibernéticos a plataformas baseadas na tecnologia Blockchain. Busca-se comprovar a inadequação das infraestruturas de redes legadas, identificar e caracterizar os atores das ameaças, suas capacidades, motivações e propósitos. São também apresentadas as melhores práticas para enfrentar essa realidade e informados cenários de aplicabilidade dessa tecnologia.

Eyal e Sirer (2013) e Courtois e Bahack (2014) estudam a vulnerabilidade da Blockchain sob a condição de ataques de mineradores desonestos, os quais conseguem receber recompensas maiores que aquelas de mineradores honestos. Usando modelagem analítica e simulação, são alcançadas evidências de que, sem regulações da mineração por mining pools, o sistema pode deixar de ser verificável de maneira descentralizada.

Karame, Androulaki e Capkun (2012), Rosenfeld (2014) e Gervais et al. (2016) estudam ataques de double-spending em sistemas baseados na Blockchain. Esses ataques objetivam permitir que um mesmo recurso seja usado em diferentes transações. A partir de modelagem analítica e simulação, são obtidos resultados que permitem inferir a probabilidade de ocorrência desses ataques e propor contramedidas de defesa.

Sompolinsky e Zohar (2015) e Karame (2016) discutem sobre a escalabilidade da Blockchain, considerando a quantidade de informações, o tempo de verificação de transações, a otimização das estruturas de dados e dos algoritmos, o tamanho dos blocos da cadeia, a diversidade de aplicações, dentre outros aspectos. Os resultados permitem conjecturar sobre uma nova geração de aplicações alicerçadas pela Blockchain.

Dorri, Kanhere e Jurdak (2017) propõem uma versão mais otimizada da Blockchain para o desenvolvimento de aplicações IoT. Essa versão tenciona diminuir o custo de processamento criptográfico, otimizar a banda e diminuir as latências de transmissão. Emprega-se um paradigma de camadas funcionais, permitindo que bases de dados locais centralizadas se comuniquem com bases públicas distribuídas. Os blocos são minerados localmente e adicionados à blockchain de sua camada, que posteriormente se torna consistente com à blockchain de camadas públicas superiores.

Danzi et al. (2017) descrevem arquiteturas gerais e protocolos de sincronização para nós de clientes IoT usando tecnologia Blockchain. Consideram-se diferentes capacidades de transmissão da rede, níveis de segurança e características intrínsecas de ambientes wireless. Nesse trabalho são modelados e caracterizados analiticamente os tráfegos gerados pelos protocolos de sincronização e, usando simulações, são avaliados o consumo de energia e o custo de banda para sincronização da blockchain.

Rodrigues (2017b) analisa a eficiência e a segurança da tecnologia Blockchain. Usando modelagem analítica e simulação, são realizados experimentos em diferentes cenários de operação. Os resultados apontam para uma promissora eficiência na manipulação de dados e uma atrativa segurança quanto à inviolabilidade da informação.

Li e Liao (2017) apresentam o protocolo GHOST, o qual propõe a utilização de uma estrutura de dados baseada em árvores em substituição à blockchain. Utilizando principalmente modelagem analítica, os autores verificam a possibilidade da redução do tempo de confirmação das transações e a otimização do nível de segurança sistêmica. Esse trabalho pode ser visto como um dos precursores da tecnologia Tangle.

Além do protocolo GHOST, há ainda algumas outras propostas que podem ser também consideradas precursoras da tecnologia Tangle como, por exemplo, os trabalhos 
de Lerner (2015), Sompolinsky e Zohar (2013) e Lewenberg, Sompolinsky e Zohar (2015). Este ultimo, em especial, propõe um modelo de criptomoeda também baseada em DAG. No entanto, o modelo difere da Tangle nos seguintes dois pontos principais: as unidades de informação são blocos de dados e não transações individuais e, ainda, o conceito de recompensa para validação de transações é aplicado.

Por fim, Popov (2017) analisa os fundamentos teóricos matemáticos da criptomoeda iota. Esses fundamentos constituem a descrição e a explicação da tecnologia Tangle, incluindo discussões sobre o número de validações que as transações devem possuir para sua aceitação, a estabilidade do sistema, as possibilidades de ataques e vulnerabilidades, e os algoritmos de seleção de transações pré-existentes. Em que pese a existência apenas de abordagem teórica, esse trabalho constitui uma das mais importantes referências da literatura sobre a análise da tecnologia Tangle.

Ante o exposto, a contribuição e a diferenciação deste artigo se revelam pelo relativo ineditismo de uma análise competitiva entre as tecnologias Blockchain e Tangle com foco em IoT, contemplando a eficiência das estruturas de dados e algoritmos, sob o viés teórico da complexidade de tempo, além da avaliação da segurança quanto à inviolabilidade das informações, sob o viés da simulação.

\section{Eficiência: Complexidade de Tempo}

Esta seção avalia a eficiência das tecnologias Blockchain e Tangle, considerando a seguinte questão de pesquisa: quais as complexidades de tempo para a execução de operações de adição de transações (ou blocos) e consulta de transações?

\subsection{Tecnologia Blockchain}

Cada bloco minerado é propagado em broadcast na rede. Em seguida, aguarda-se que nós completos, peers da rede que mantêm uma cópia local de toda a cadeia de blocos, realizem a sua adição. A cópia da cadeia de blocos local de cada nó completo é atualizada à medida que novos blocos são minerados e propagados. A convergência das cópias locais dos nós completos ocorre conforme as informações são disseminadas.

Para adição de um novo bloco, $B_{n o v o}$, um nó completo, $P$, inicialmente examina o cabeçalho desse bloco para conhecer o valor do campo hash do bloco anterior, que é a referência para o bloco pai. Seja $h\left(B_{\text {anterior }}\right)$ este valor. Admita que $P$ possui $m$ blocos na sua cadeia local, sendo $B_{m}$ o último bloco adicionado, e $h\left(B_{m}\right)$ o hash do seu cabeçalho.

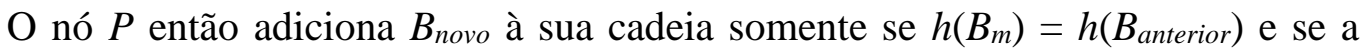
prova de trabalho tiver sido realizada, ou seja, se o valor do nonce tiver sido determinado. Embora a computação do valor de nonce seja intrincada, a sua verificação é de complexidade constante, pois basta que o valor de nonce seja testado. Assim, a complexidade de tempo de adição de um bloco é de apenas $O(1)$. No entanto, é preciso ressaltar que, para que a adição ocorra, é necessário que o bloco já tenha sido minerado. Ou seja, existe um custo computacional anterior que, na prática, não pode ser ignorado.

Para consultar sobre a existência de uma transação em um bloco no sistema são usados nós especiais da rede, chamados de nós de Verificação Simplificada de Transação (VST). Esses nós utilizam o chamado caminho de Merkle ou caminho de autenticação [Antonopoulos 2017], conforme explicado a seguir. 
Seja $Q$ um nó VST que deseja consultar a existência da transação $s$, tendo como uma das partes envolvidas um cliente de endereço lógico $E$. Esse nó $Q$ deve então criar um filtro em suas conexões com os demais peers da rede para restringir as transações a ele informadas, ou seja, apenas blocos com transações que se relacionam ao endereço $E$ devem ser informados a ele. Assim, quando um peer da rede vê um bloco com uma transação de endereço $E$, ele então informa sobre aquele bloco ao nó $Q$. Para tanto, ele envia uma mensagem especial, denominada mensagem de bloco de Merkle (MBM). Essa mensagem contém o cabeçalho do bloco e o caminho de Merkle que liga a transação $s$ à raiz da árvore de Merkle do bloco.

A árvore de Merkle [Berman; Karpinski; e Nekrich 2007], conhecida como árvore binária de hash, é construída a partir dos seus vértices folhas. Cada folha guarda o hash de uma das transações existentes no bloco. São então computados recursivamente os hashes de pares de vértices de mesmo nível (i.e., vértices irmãos), a partir dos vértices folhas em direção à raiz, até que se obtenha um único hash, o qual constitui o resumo de todas as transações existentes no bloco. Esse resumo é guardado em um campo do cabeçalho do bloco, denominado raiz da árvore de Merkle. No caso de o número de transações ser ímpar, a última transação é duplicada, obtendo sempre uma árvore binária cheia [Cormen; Leiserson; e Rivest, 2009].

Seja então a sequência de vértices $\left(v_{1}, v_{2}, \ldots, v_{l}\right)$ o caminho de Merkle informado ao nó $Q$. Cada vértice da sequência está em um nível imediatamente inferior ao anterior na árvore de Merkle. Mais especificamente, o vértice $v_{1}$ é uma folha e o vértice $v_{l}$ está no nível imediatamente superior ao nível da raiz da árvore, que está no nível 1. Além disso, o vértice $v_{1}$ é irmão do vértice folha que armazena o hash da transação $s$.

A consulta da transação $s$ consiste então em computar recursivamente o hash de vértices irmãos correspondentes na árvore em diferentes níveis sucessivos, a partir do vértice folha $v_{1}$ em direção à raiz. Ao atingir-se o segundo nível, é então verificado se o hash computado é igual ao valor da raiz, obtido do exame do cabeçalho do bloco, que é também informado na MBM. Se ocorre a identidade, então a transação $s$ de fato existe.

Essa operação de consulta tem complexidade de tempo $O\left(\left\lfloor\log _{2}(2 n-1)\right\rfloor\right)$, onde $n$ é o número total de transações existentes no bloco. Esse resultado é bastante eficiente por ser logarítmico em função da quantidade de dados. Isso ocorre porque a árvore de Merkle construída é sempre uma árvore binária cheia e, portanto, goza da propriedade de altura logarítmica [Cormen; Leiserson; e Rivest 2009].

\subsection{Tecnologia Tangle}

Como mencionado, cada nó do DAG armazena uma única transação. Considerando nós VST, a consulta de uma transação $s$ pode então ser realizada em tempo constante $O(1)$, dado que se saiba qual o nó que armazena a transação $s$. Em termos de complexidade de tempo, tem-se então que Tangle é mais eficiente que Blockchain para realizar consultas, pois a complexidade desta última é maior, sendo igual a $O\left(\log _{2}(2 n-1) \mathrm{J}\right)$, onde $n$ é o número total de transações existentes no bloco (vide Subseção 4.1).

Com relação à adição de uma transação $s$ na Tangle, tem-se a análise baseada nos dois pontos a seguir. Primeiro, é preciso realizar um processamento matemático para resolução de um hash criptográfico, i.e., obtenção do valor nonce. Esse hash 
criptográfico envolve metadados relacionados à identificação da transação $s$, além da transação $s$ propriamente dita. Seja $C_{\mathrm{T}}$ a complexidade dessa operação.

Segundo, é preciso validar duas transações pré-existentes no sistema. O processo de validação é simples, consistindo apenas em verificar se há conflitos entre as transações e se a prova de trabalho foi realizada pelo respectivo nó da transação a ser validada. Essa verificação pode ser feita em $O(1)$. Resulta então que a complexidade final de adição é $C_{\mathrm{T}}+O(1)=C_{\mathrm{T}}$, ou seja, é determinada pela complexidade da realização da prova de trabalho.

Para fins de comparação, tem-se que a adição de um bloco é feita em $O(1)$ na tecnologia Blockchain. Na prática há, contudo, a necessidade da mineração prévia do bloco a ser adicionado (vide Subseção 4.1). Seja o custo dessa mineração igual a $C_{\mathrm{B}}$. O custo final de adição na Blockchain é, portanto, $C_{\mathrm{B}}+O(1)=C_{\mathrm{B}}$.

Tendo em vista que a mineração de um bloco na Blockchain envolve um hash criptográfico com metadados relacionados à identificação de todo o bloco, além de todas as transações nele existentes, tem-se que $C_{\mathrm{B}}>C_{\mathrm{T}}$. Ou seja, a tecnologia Tangle resulta mais eficiente que a tecnologia Blockchain quanto à complexidade de tempo da adição. Essa conclusão é comparativa e independe dos valores absolutos de $C_{\mathrm{B}}$ e $C_{\mathrm{T}}$.

Como observação final, deve-se mencionar que cada adição na Blockchain considera $n$ transações de uma só vez, e que cada adição na Tangle se refere a apenas uma única transação. Mesmo assim, é possível conjecturar que $C_{\mathrm{B}} / n>C_{\mathrm{T}}$ em virtude da natureza do cálculo de hash envolvido [Cormen; Leiserson; e Rivest 2009].

\section{Segurança: Inviolabilidade da Informação}

Esta seção avalia a segurança das tecnologias Blockchain e Tangle, considerando a seguinte questão de pesquisa: qual o tempo de registro de uma transação no sistema para garantir um nível de segurança aceitável, significando inviolabilidade da informação?

\subsection{Descrição dos Cenários de Investigação}

Para entender a questão de pesquisa na Blockchain, considere o cenário descrito a seguir [Nakamoto 2008]. Quando dois mineradores distintos enviam diferentes versões daquele que seria o próximo bloco da blockchain, os nós completos vão receber uma ou outra versão primeiramente. Cada nó completo considera a primeira versão recebida, mas vai também criar uma ramificação em sua blockchain local para adicionar a outra versão do bloco recebida posteriormente. Passam a existir duas ramificações: uma para a primeira versão e a outra para a segunda versão.

A seleção entre as duas ramificações ocorre quando as próximas provas de trabalho são encontradas, fazendo com que uma das ramificações se torne mais longa que a outra. Neste instante, a ramificação mais curta é desprezada. Para ser considerada válida, uma transação deve então pertencer à ramificação mais longa.

A competição entre duas ramificações pode ser modelada como uma corrida para adição de blocos à blockchain, como explicado a seguir [Nakamoto 2008]. Um minerador desonesto deseja realizar uma fraude, substituindo um bloco $B$ legítimo adicionado por um minerador honesto. Para isso, o minerador desonesto espera até que $z$ blocos subsequentes a $B$ sejam adicionados à blockchain. Neste instante, o minerador 
honesto está minerando o bloco $z+1$. Explica-se que essa espera de $z$ blocos é o tempo que o cliente da aplicação tolera esperar para ter sua transação confirmada no sistema.

O minerador desonesto então inicia a mineração explícita de blocos e tenta ser mais rápido que o minerador honesto para compensar a desvantagem de $z$ blocos, fazendo sua ramificação se tornar mais longa que a ramificação contendo o bloco legítimo $B$. Um detalhe importante é que o minerador desonesto minera blocos secretamente em taxa compatível com sua capacidade para que, ao iniciar a competição explícita com o minerador honesto, já possua blocos minerados para inserir no sistema.

Iniciada a competição, tem-se o seguinte modelo parametrizado [Nakamoto 2008]: com probabilidade $p$, o próximo bloco minerado da blockchain é do minerador honesto, e com probabilidade $q=(1-p)$, o próximo bloco minerado é do minerador desonesto. A chegada de blocos minerados à blockchain segue um processo de Poisson com parâmetro $\mu$. Esse modelo permite estimar a probabilidade de fraude para um dado valor de $z$. Quanto maior é $z$, menor é a probabilidade de fraude e maior é o tempo de espera (i.e., tempo de registro da transação). Ou seja, há um compromisso entre a segurança e o tempo de espera. Esse modelo é resolvido por simulação na Subseção 5.2.

O cenário de investigação da Tangle é análogo ao da Blockchain [Popov 2017]. A competição, porém, é pelo número de validações da transação. $\mathrm{O}$ nó fraudador deseja substituir uma transação legítima $T$ do DAG. Ele então espera até que a transação $T$ atinja $v$ validações, que reflete o tempo que o cliente da aplicação tolera esperar para ter a transação confirmada. Em seguida, ele insere uma transação fraudulenta $F$ no DAG para tentar substituir a transação $T$. Para isso, ele passa a criar novas transações que só validam a transação $F$. O objetivo é que, eventualmente, a transação $F$ passe a ter mais validações que a transação $T$. Como na Blockchain, o fraudador na Tangle trabalha secretamente em taxa compatível com sua capacidade para que, ao iniciar a competição explícita, ele já possua transações prontas para inserir no DAG.

O modelo parametrizado a seguir é usado para análise da Tangle [Popov 2017]: a chegada de transações ao DAG segue um processo de Poisson com parâmetro $\beta$; a fração de transações que valida a transação legítima $T$ é $k \%$ do total de transações, e a fração de transações que valida a transação fraudulenta $F$ é $r \%$ do total de transações, sendo $k+r=100$. Esse modelo permite calcular a probabilidade de fraude para um dado valor de $v$. Quanto maior é $v$, menor é a probabilidade de fraude e maior é o tempo de espera. Ou seja, como na Blockchain, há um compromisso entre a segurança e o tempo de espera. Esse modelo é resolvido por simulação na Subseção 5.2.

\subsection{Ambiente de Simulação e Modelos}

A simulação usa o Tangram-II [De Souza e Silva; Figueiredo; e Leão 2009]. Tangram-II é um ambiente de modelagem de sistemas computacionais concebido na Universidade Federal do Rio de Janeiro, com a participação da Universidade da Califórnia em Los Angeles nos EUA. Os modelos são definidos em termos de objetos que interagem entre si por mensagens, podendo ser resolvidos analiticamente ou via simulação.

O modelo de simulação da Blockchain é explicado a seguir. São criados dois objetos: $O b j_{1}$ e $O b j_{2}$. O objeto Obj $j_{1}$ emula os mineradores (honestos e desonestos) do sistema, os quais têm individualmente a mesma capacidade de mineração. O processo de Poisson tem seu parâmetro $\mu$ igual a um bloco minerado a cada 10 minutos, 
conforme definição da Blockchain [Rodrigues 2017a]. O objeto $\mathrm{Obj}_{2}$ emula a blockchain do sistema, permitindo monitorar a diferença de blocos entre as ramificações honestas e desonestas. O sistema é considerado no estado estacionário.

O modelo de simulação da tecnologia Tangle é explicado a seguir. São criados três objetos: $O b j_{1}, O_{b j}$ e $O b j_{3}$. Os dois primeiros objetos emulam os nós honestos e desonestos, respectivamente, os quais têm individualmente a mesma capacidade de processamento. Os nós honestos coletivamente contribuem com $k \%$ das transações totais do sistema, e os desonestos com $r \%$ das transações totais, sendo $k+r=100$. $\mathrm{O}$ processo de Poisson tem seu parâmetro $\beta$ igual a 500 transações a cada 10 minutos. Esse valor permite uma comparação mais equânime com a tecnologia Blockchain, pois o número médio de transações por bloco é 500 [Antonopoulos 2017]. O objeto $\mathrm{Obj}_{3}$ emula o DAG, permitindo monitorar a diferença de validações entre as transações honestas e desonestas. O sistema é considerado no estado estacionário.

Cabe destacar que os resultados de simulação têm intervalos de confiança de 95\% que estão dentro do limite de 5\% dos valores estimados, tendo sido consideradas 30 execuções (rodadas) com um tempo de simulação de 21.000 minutos cada. Estes resultados e as correspondentes análises estão na subseção seguinte. Por restrição de espaço, menciona-se que apenas os resultados mais relevantes para suporte às conclusões atingidas são apresentados no que se segue.

\subsection{Resultados e Análises}

Os resultados obtidos para Blockchain estão nas Figuras 1 e 2. A Figura 1 traz resultados de seis cenários de análise: $S_{1}, S_{2}, S_{3}, S_{4}, S_{5}$ e $S_{6}$. Nos três primeiros cenários, considera-se $p=0,6$ e varia-se $z$ para determinar a probabilidade de a diferença de tamanho entre as ramificações concorrentes exceder o valor de $X$ blocos. Nos três cenários seguintes, repete-se a análise para $p=0,8$.

A partir dos resultados dessa figura, conclui-se que maiores valores de $p$ e $z$ tendem a fornecer maior segurança, pois esses valores tendem a aumentar a diferença de tamanho entre as ramificações. Por exemplo, a maior probabilidade associada a $X=0$ é obtida para $p=0,8$ e $z=6$ (Cenário $S_{6}$ ). Isso significa que a diferença de tamanho neste cenário tem mais chance de ser maior do que zero do que nos demais cenários. Essa superioridade se mantém destacada para todos os valores de $X$ em que a curva está definida. Assim, a fraude tem menor chance de ocorrer, pois apenas existiria se as ramificações se igualassem, compensando a desvantagem inicial de $z$ blocos.

Por outro lado, aumentos arbitrários de $p$ e $z$, sem prévia avaliação, podem ser inócuos. Por exemplo, os resultados obtidos para os Cenários $S_{1}$ e $S_{2}$, respectivamente, são praticamente idênticos, ou seja, o aumento de $z$, de 1 para 3 , pouco contribui para segurança quando $p$ é mantido em 0,6 . Diferentemente, quando $p=0,8$, o aumento de $z$, também de 1 para 3 , aumenta a segurança do sistema, pois a diferença entre as ramificações se torna maior, como visto nos resultados dos Cenários $S_{4}$ e $S_{5}$.

A Figura 2, por sua vez, apresenta resultados para os mesmos seis cenários anteriores. Porém, agora são destacados valores médios das diferenças de tamanhos entre as ramificações. Confirma-se novamente que maiores valores de $p$ e $z$ tendem a ocasionar uma maior segurança. Por exemplo, a diferença registrada no Cenário $S_{6}$ é quase cinco vezes maior que aquela do Cenário $S_{1}$. Porém, como antes, também se 
confirma que aumentos arbitrários de $p$ e $z$ podem resultar inócuos. Por exemplo, nos Cenários $S_{5}$ e $S_{6}$, dobrar o valor de $z$, de 3 para 6 , não faz a diferença média ser dobrada. Enfim, como diretriz de projeto, sugere-se admitir valores de $p$ acima de 0,5 e valores de $z$ que devem observar a tolerância do cliente da aplicação para espera de confirmação.

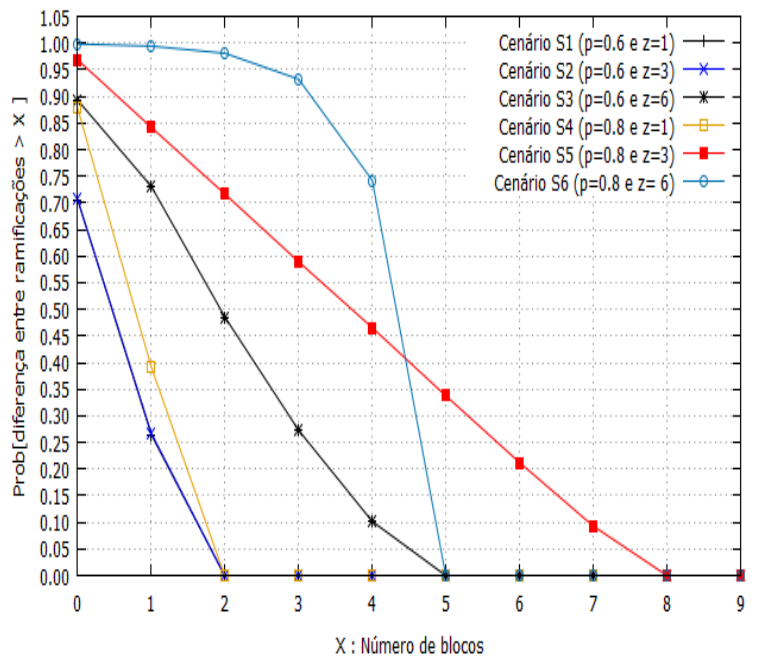

Figura 1. Diferença entre ramificações.

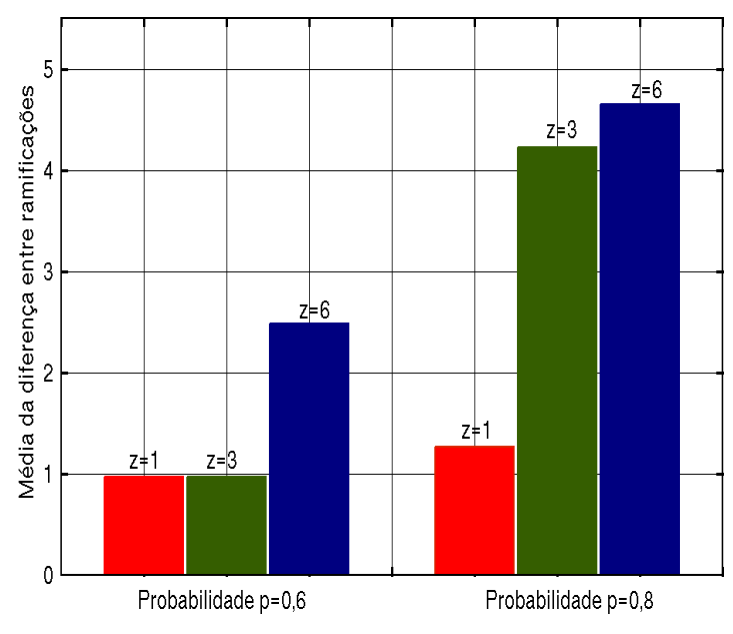

Figura 2. Média da diferença.

As Figuras 3 e 4, por sua vez, trazem os resultados para Tangle. São considerados seis cenários de análise: $S_{7}, S_{8}, S_{9}, S_{10}, S_{11}$ e $S_{12}$. Considere inicialmente a Figura 3. Nos três primeiros cenários, tem-se $k=60 \%$ e $v$ sendo variado para determinar a probabilidade de a diferença de validações entre as transações $T$ e $F$ exceder um valor $Y$. Nos três cenários seguintes, faz-se a mesma análise para $k=80 \%$. Considere agora a Figura 4. Nesta figura são destacados os valores médios das diferenças de validações entre as transações $T$ e $F$ para os mesmos seis cenários anteriores.

A partir dos resultados da Figura 3, observa-se que maiores valores de $k$ e $v$ redundam em maior segurança. Isso também ocorre na Blockchain ao aumentar-se os valores de $p$ e $z$. Entretanto, na Tangle esse aumento de segurança é mais contundente. Por exemplo, vê-se que as curvas de probabilidade têm valor inicial mais próximo ao valor unitário que aquelas da Figura 1. Além disso, mesmo aumentos independentes de $v$ ou de $k$, sem avaliação prévia, são capazes de aumentar as probabilidades. Essa independência entre parâmetros não foi verificada na Blockchain. Ou seja, o nível de segurança é mais facilmente otimizado na Tangle.

$\mathrm{Na}$ Figura 4, por sua vez, tem-se que, para $k=60 \%$ ou $80 \%$, ao dobrar-se ou triplicar-se o valor de $v$, há um aumento de mesma magnitude nos correspondentes valores médios das diferenças de validações entre as transações $T$ e $F$, implicando maior segurança. Essa correspondência é diferente daquela observada na Blockchain. Em síntese, a tecnologia Tangle é uma solução mais atrativa que Blockchain. Por fim, como diretriz de projeto, sugere-se admitir valores de $k$ acima de $50 \%$ e valores de $v$ que devem observar a tolerância do cliente da aplicação para espera de confirmação. 


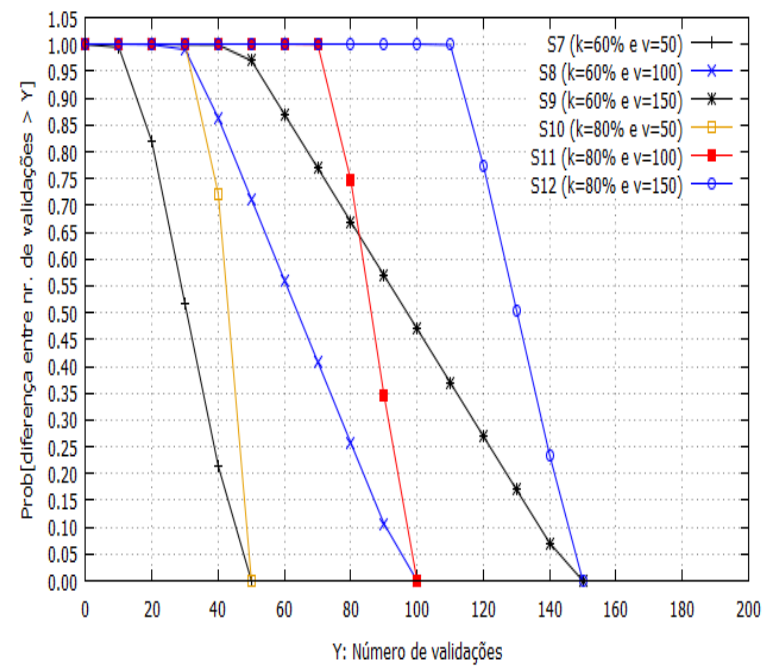

Figura 3. Diferença entre validações.

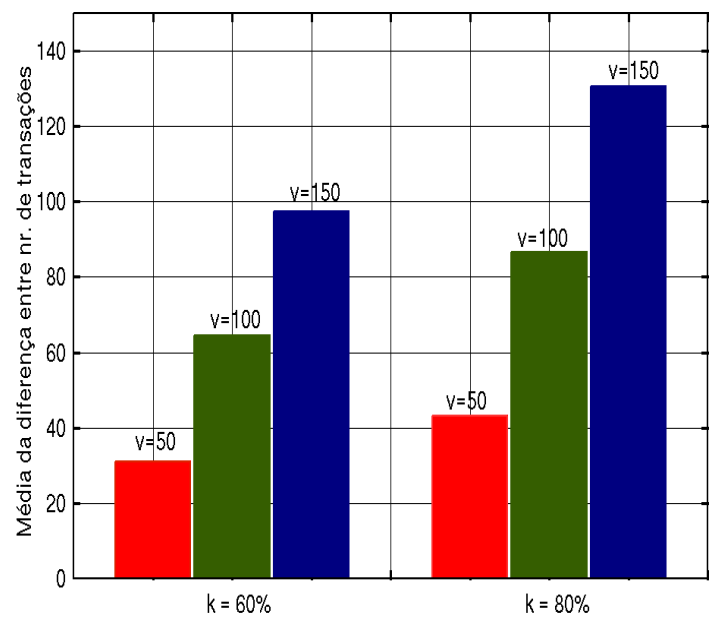

Figura 4. Média da diferença.

\section{Conclusões e Trabalhos Futuros}

Este artigo realizou uma análise competitiva entre as tecnologias Blockchain e Tangle. Para tanto, inicialmente foi feito um estudo teórico das estruturas de dados e dos algoritmos para manipulação das informações. Na sequência, por meio de simulação, foram examinados diferentes cenários para mensurar o nível de segurança do sistema.

O estudo teórico mostrou principalmente que o uso da árvore de Merkle para Blockchain é uma solução teórica efetiva, pois garante a otimização da complexidade de tempo para execução de consultas e adições de transações. Essa efetividade se mostra promissora na prática conforme sejam maiores os blocos de dados. O contraponto é que o processamento matemático da prova de trabalho demanda significativa capacidade de processamento, justamente por lidar com o bloco inteiro de informações.

Por sua vez, o uso de DAG para Tangle teve seu destaque pela sua simplicidade de implementação: uma transação por vértice. Isso possibilita uma execução prática otimizada de consultas e adições de transações. Além disso, a realização da prova de trabalho é menos onerosa que aquela da Blockchain por lidar com menos informações. Dado que o processamento típico em cenários de IoT envolve poucos dados, a Tangle termina sendo então uma tecnologia mais atrativa na prática que a Blockchain.

Em relação à segurança, tem-se precipuamente o que se segue. Os experimentos permitiram ver que as tecnologias Blockchain e Tangle apresentam satisfatório nível de segurança no que se refere à inviolabilidade das informações, podendo ser consideradas para projetos reais de aplicações IoT. No entanto, sob o viés da competição, a Tangle foi entendida aqui como uma solução mais atrativa. Essa conclusão adveio do fato de ela ser uma solução que mais objetivamente e facilmente responde à mudança de configuração de parâmetros para alcançar o nível de segurança desejado.

Como trabalhos futuros, tem-se: (i) análise de cenários mais complexos, com diferentes valores de validação de transações na Tangle e com disputas entre múltiplas ramificações na Blockchain; (ii) desenvolvimento de modelos analíticos e novos modelos de simulação com distribuições diferentes de Poisson; e (iii) análise competitiva dos algoritmos de consenso proof of work e proof of stake [Li e Liao 2017]. 


\section{Referências}

Antonopoulos, M. (2017). Mastering Bitcoin: Programming the Open Blockchain. 2nd Edition. Sebastopol, California: O’Reilly Media.

Berman, P.; Karpinski, M.; and Nekrich, Y. (2007). Optimal trade-off for Merkle tree traversal. Theoretical Computer Science, v. 372, n. 1, pp. 26-36.

Chandrana, N. R. and Manuelb, E. M. (2016). Performance Analysis of Modified SHA-3. Procedia Technology, v. 24, pp. 904-910.

Cormen, T. H; Leiserson, C. E.; and Rivest, R. L. (2009). Introduction to Algorithms. 3rd Edition. Cambridge, Massachussets: MIT Press.

Courtois, N. T. and Bahack, L. (2014). On subversive miner strategies and block withholding attacks in bitcoin digital currency. CoRR, abs/1402.1718. Disponível em: https://arxiv.org/abs/1402.1718. Acessado em: 8 de dezembro de 2017.

Danzi, P. et al. (2017). Analysis of the Communication Traffic for Blockchain Synchronization of IoT Devices. CoRR, abs/1711.00540. Disponível em: https://arxiv.org/abs/1711.00540v1. Acessado em: 8 de dezembro, 2017.

De Souza e Silva, E.; Figueiredo, R.; and Leão, R. (2009). The TANGRAM-II integrated modeling environment for computer systems and networks. ACM SIGMETRICS Performance Evaluation Review, v. 36, n. 4, pp. 64-69.

Dorri, A.; Kanhere, S.; and Jurdak, R. (2017). Towards an Optimized Blockchain for IoT. In: International Conference on Internet-of-Things Design and Implementation (IoTDI'17), Pittsburgh, PA, USA.

Eyal, I. and Sirer, E. G. (2014). Majority is not enough: bitcoin mining is vulnerable. In: International conference on financial cryptography and data security, pp. 436-452. Springer, Berlin, Heidelberg.

Garay, J.; Kiayias, A.; Leonardos, N. (2015). The bitcoin backbone protocol: analysis and applications. LNCS, Springer, Berlin, Heidelberg, v. 9057, pp. 281-310.

Gaur, A. et al. (2015). Smart city architecture and its applications based on IoT. Procedia Computer Science, v. 52, p. 1089-1094.

Gervais, A. et al. (2016). On the security and performance of proof of work blockchains. In: ACM Conference on Computer and Communications Security, Vienna, Austria.

Gilbert, H. and Handschuh, H. (2004). Security analysis of SHA-256 and sisters. LNCS, Springer, Berlin, Heidelberg, v. 3006, pp. 175-193.

Hassanalieragh, M. et al. (2015). Health monitoring and management using Internet-ofThings (IoT) sensing with cloud-based processing: Opportunities and challenges. In: IEEE International Conference on Services Computing, p. 285-292.

Huckle, S. et al. (2016). Internet of Things, blockchain and shared economy applications. Procedia Computer Science, v. 98, pp. 461-466.

IOTA. (2017). What is IOTA? Disponível em: https://iota.readme.io/docs. Acessado em: 8 de dezembro de 2017. 
Karame, G. O. (2016). On the security and scalability of bitcoin's blockchain. In: ACM Conference on Computer and Communications Security (CCS'16), Vienna, Austria.

Karame, G. O.; Androulaki, E.; Capkun, S. (2012). Two bitcoins at the price of one? Double-spending on fast payments in bitcoin. In: ACM Conference on Computer and Communications Security (CCS'12), Raleigh, NC, USA.

Lerner, S. D. (2015). DagCoin: a cryptocurrency without blocks. Disponível em: https://bitslog.files.wordpress.com/2015/09/dagcoin-v41.pdf. Acessado em: 6 de dezembro de 2017.

Lewenberg, Y.; Sompolinsky, Y.; and Zohar, A. (2015). Inclusive Block Chain Protocols. Disponível em: http://fc15.ifca.ai/preproceedings/paper_101.pdf. Acessado em: 8 de dezembro de 2017.

Li, I-C and Liao, T-C. (2017). A Survey of blockchain security issues and challenges. International Journal of Network Security, v. 19, n. 5, pp.653-659, 2017.

Nakamoto, S. (2008). Bitcoin: A peer-to-peer electronic cash system. Disponível em: https://bitcoin.org/bitcoin.pdf . Acessado em: 8 de dezembro de 2017.

Opara, E. U. and Soluade, O. A. (2015). Straddling the next cyber frontier: the empirical analysis on network security, exploits, and vulnerabilities. International Journal of Electronics and Information Engineering, v. 3, n. 1, pp. 10-18.

Popov, S. (2017). The Tangle - Version 1.3. Disponível em: https://iota.org/IOTA_Whitepaper.pdf. Acessado em: 6 de dezembro, 2017.

Rodrigues, C. K. S. (2017a). Sistema Bitcoin: uma análise da segurança das transações. Revista Brasileira de Sistemas de Informação, v. 10, n. 3, pp. 5-23.

Rodrigues, C. K. S. (2017b). Uma análise simples de eficiência e segurança da tecnologia Blockchain. Revista de Sistemas e Computação, v. 7, n. 2, pp. 147-162.

Rosenfeld, M. (2014). Analysis of hasrate-based double spending. CoRR, abs/1402.2009. Disponível em: https://arxiv.org/abs/1402.2009 . Acessado em: 8 de dezembro de 2017.

Silva, G. A. e Rodrigues, C. K. S. (2016). Mineração individual de bitcoins e litecoins no mundo. In: Simpósio Brasileiro em Segurança da Informação e de Sistemas Computacionais (SBSeg 2016), Niterói, Rio de Janeiro, Brasil.

Singh, J. (2014). Cyber attacks in cloud computing: a case study. International Journal of Electronics and Information Engineering, v. 1, n. 2, pp. 78-87.

Sompolinsky, Y. and Zohar, A. (2013). Accelerating Bitcoin's Transaction Processing Fast Money Grows on Trees, Not Chains. Cryptology ePrint Archive, Report 2013/881. Disponível em: https://eprint.iacr.org/2013/881. Acessado em: 8 de dezembro de 2017.

Sompolinsky, Y. and Zohar, A. (2015). Secure high-rate transaction processing in bitcoin. LNCS, Springer, Berlin, Heidelberg, v. 8975, pp. 507-527.

Sonstebo, D. (2017). Curl disclosure, beyond the headline. Disponível em: https://blog.iota.org/curl-disclosure-beyond-the-headline-1814048d08ef. Acessado em: 6 de dezembro de 2017. 